\title{
Phase Transitions of Hard Sphere Lattice Gases ${ }^{\star}$
}

\author{
L. K. Runnels \\ Department of Chemistry, Louisiana State University, Baton Rouge, Louisiana, USA
}

Received May 16, 1974

\begin{abstract}
A unified proof is given for the existence of a phase transition for any two or three dimensional lattice gas with hard cores excluding nearest neighbor occupancy, provided only that the lattice is an open one (possessing two sublattices). It is not required that one sublattice be a translate of the other. Consequently the proof applies to the plane hexagonal and to the diamond lattices, as well as the "cubic" lattices previously proved to have phase transitions. The models are converted to equivalent Ising spin 1/2 systems on one sublattice by a "partial trace" over the other. The spin system has many-spin interactions including some of odd order, which destroys up-down symmetry, but recent work of Pirogov and Sinai on such systems is shown to be applicable and to prove the existence of the phase transition.
\end{abstract}

\section{Introduction}

One of the most useful techniques for the rigorous proof of the existence of phase transitions in model systems has been the "contour" method originally due to Peierls [1]. The proof consists in showing that boundary conditions determine the equilibrium state throughout an arbitrarily large system - under conditions of sufficiently large interaction potentials (or sufficiently low temperature). Thus the boundary conditions determine the sign of the magnetization of an Ising system, the density of an attractive lattice gas, or the relative concentrations in a multicomponent system.

In each of these cases the proof succeeds only on a portion of some "symmetry line" appropriate to the model, such as zero magnetic field in the Ising case. For the attractive lattice gas the corresponding symmetry requirement is that the chemical potential (one-body energy) just balance the pair interactions in the completely filled lattice. For the multicomponent Widom-Rowlinson model the symmetry condition is that the chemical potentials of all components be equal [2]. In each case the symmetry condition insures that there is no term in the Hamiltonian proportional to volume that favors any of the competing equilibrium

\footnotetext{
* Supported in part by National Science Foundation Grant No. GP 33535X.
} 
states - leaving only the boundary, or surface, terms to prevail under conditions promoting long range order.

A somewhat different application of the Peierls technique was used [3] to demonstrate the existence of the phase transition for lattice gases with repulsive (including hard core) interactions. In these cases the competing equilibrium states correspond to the filling of different sublattices. The underlying symmetry condition in these cases is that the chemical potential is the same for a particle on either sublattice, so that at large values of the chemical potential the sublattice occupied is determined again by the boundary conditions. The proofs have succeeded only in cases where the sublattices are translates of each other - square, cubic, triangular [4]. These proofs fail in cases of lower symmetry - hexagonal and diamond lattices - although numerical studies $[5,6]$ show little qualitative difference between the two classes of lattices.

Recently Pirogov and Sinai [7] have provided a significant extension of the theory to more general interactions in which the "symmetry line" is not apparent intuitively. In the language of the Ising model this generalization meant that the spin interactions were no longer restricted to interactions of even order, with attendant symmetry of positive and negative magnetizations. The overall interaction must still be essentially ferromagnetic, however, in a sense to be made more precise below.

It was then shown that the phase transition of binary lattice gases (of the Widom-Rowlinson type) could be interpreted in terms of the Pirogov-Sinai development [8]. The technique was first to transform the binary lattice gas to an equivalent one-component lattice gas with many-body interactions (through partial summation over configurations of particles of one type). Then this interacting lattice gas was transformed in the standard way to an equivalent spin system. The final step was to show that the conditions of the Pirogov-Sinai theorem are met.

The purpose of this article is to show that the same sort of interpretation can be applied to hard core lattice gases, provided the hard core extends only to nearest neighbors and the lattice possesses exactly two sublattices. This includes the "cubic" lattices (any number of dimensions greater than one) already proven to have phase transitions, but also the hexagonal and diamond lattices for which proofs have been lacking. The body-centered cubic lattice may also be treated by either approach.

The transformation technique is a lattice gas version of the "partial trace method" used by Gruber and Merlini [9] for spin 1/2 Ising systems. The partial trace method is a generalization of the "star-triangle" and the "decoration" transformations [10]. Indeed Widom and coworkers employed a lattice gas version of the decoration transformation to study special models of multicomponent lattice gases and to reduce them to equivalent ordinary lattice gases with nearest neighbor attractions [11]. 


\section{The Pirogov-Sinai Theorem}

Due to the central role it plays in our development, we wish to state here the theorem of Pirogov and Sinai [7]. The theorem deals with Ising spin systems on $d$-dimensional cubic lattices, $d \geqq 2$. We write the Hamiltonian as

$$
H_{\Lambda}=-\sum_{P \subset A} J_{P} \sigma_{P}
$$

where $\Lambda$ is a finite lattice,

$$
\sigma_{P}=\prod_{x \in P} \sigma_{x}
$$

and $\sigma_{x}= \pm 1$. A one-body term $J_{\{x\}}$ is the magnetic field $h$, assumed homogeneous. The two-body terms $J_{P},|P|=2$, are assumed to be nonnegative; moreover, they are strictly positive (ferromagnetic) for $P=\{x, y\}$ with $x$ and $y$ being nearest neighbor sites. The number of sites in set $P$ is denoted $|P|$. The interactions $J_{P}$ have finite range and thus vanish if $P$ has sufficiently large diameter.

We call $H_{\Lambda}(+)$ and $H_{\Lambda}(-)$ the energies when all spins are +1 and -1 , respectively. The equation

$$
\begin{aligned}
H_{\Lambda}(+) & =-h|\Lambda|-\sum_{\substack{P \subset A \\
|P| \geqq 2}} J_{P} \\
& =h|\Lambda|-\sum_{\substack{P \subset A \\
|P| \geqq 2}}(-)^{|P|} J_{P} \\
& =H_{\Lambda}(-)
\end{aligned}
$$

can always be solved for the magnetic field. We call the solution $h^{*}$; it is the magnetic field in which the $(+)$ configuration and the $(-)$ configuration have the same energy, to be denoted $H_{A}( \pm)$. For the special case of only nearest neighbor interactions $h^{*}=0$. In general $h^{*}$ can be $\Lambda$-dependent, although with periodic couplings this will not occur (see Section IV).

Any spin configuration defines a set of closed contours in the usual way: we draw a contour segment of lattice unit length as the perpendicular bisector of each line joining unlike nearest neighbor spins. The closed contours are the union of the segments, with the understanding that closed cycles no more distant from each other than twice the range of the interaction are parts of the same contour.

We consider first a configuration $\alpha_{0}$ which produces only one contour $\gamma$. We require the outer layers of $\Lambda$ to consist of all $+(-)$ spins. The natural definition of the contour energy of $\gamma$ in the field $h^{*}$ is its energy relative to the $+(-)$ configuration:

$$
W_{\gamma}^{ \pm}=H_{\Lambda}\left(\alpha_{0}\right)-H_{\Lambda}( \pm)
$$

and we notice that $W_{\gamma}^{+}$is not necessarily equal to $W_{\gamma}^{-}$. Now if a configuration $\alpha$ produces the family $\Gamma$ of contours as defined above, we can write 
the total energy of $\alpha$ in the field $h^{*}$ with boundary spins $+(-)$ as

$$
H_{\Lambda}(\alpha)-H_{\Lambda}( \pm)=\sum_{\gamma \in \Gamma} W_{\gamma}^{ \pm} \text {. }
$$

In the family $\Gamma$ the + or - symbol in $W_{\gamma}^{ \pm}$for outer contours (those which can be connected to the boundary by a path not intersecting $\gamma$ ) is that of all the boundary spins. Moving inward in a nested set of contours the symbols alternate.

We can give an alternate and more pictorial description of the contour energies in the field $h^{*}$. Remembering that the interactions have finite range (and are assumed translationally invariant) we can write

$$
H_{\Lambda}=\sum_{x \in \Lambda} U_{E_{x}}^{\prime}(\alpha)
$$

where $U_{E_{x}}^{\prime}(\alpha)$ is an appropriately defined energy of the finite set $E_{x}$, which is indexed by a reference site $x$. In order to avoid overcounting some interactions, we arrive at the definition

$$
U^{\prime}{ }_{E_{x}}=-\sum_{P \subset E_{x}} J_{P} \sigma_{P} / v_{P}
$$

where $v_{P}$ is the number of translates of set $P$ contained in $E_{x}$. The sets $E_{x}$ are not uniquely defined, but greater convenience will result from smaller sets which must, however, be large enough to contain prototypes of all interactions.

Finally we set

$$
U_{E_{x}}=U_{-E_{x}}^{\prime}-U_{E_{x}}^{\prime}( \pm)
$$

where $U_{E_{x}}^{\prime}( \pm)$ is the energy of the finite set $E_{x}$ when all contained spins have the same value and the external field has the value $h^{*}$. Now we may express the energy of contour $\gamma$, produced by configuration $\alpha$ as

$$
W_{\gamma}^{ \pm}=\sum_{E_{x}: E_{x} \cap \gamma \neq 0} U_{E_{x}}(\alpha) \text {. }
$$

We say that $\gamma$ intersects $E_{x}$ if $\gamma$ contains a contour segment bisecting two sites of $E_{x}$. The sign + or - in $W_{\gamma}{ }^{ \pm}$is determined by the spins at the periphery of $\Lambda$ in the configuration $\alpha$. We allow only configurations which have "pure" boundaries - all + or all - . Each contour is thus "paved" with all finite sets $E_{x}$ which intersect it. Due to the definitions of the contours and the sets $E_{x}$, no set paves contour segments of more than one contour, although a set may well cover more than one segment of the same contour.

We are now in a position to state the Pirogov-Sinai theorem. As usual $\beta=1 / k T$ is proportional to the inverse temperature, and $|\gamma|$ is the number of contour segments in $\gamma$.

Theorem. For a spin system with interactions as described above, suppose $W_{\gamma}{ }^{ \pm}>d|\gamma|$ for any contour in the field $h^{*}$ with pure boundary 
conditions for some constant $d>0$. Then there is a $\beta_{0}, 0<\beta_{0}<\infty$, such that for $\beta>\beta_{0}$ there is a magnetic field $h=h(\beta)$ at which a phase transition occurs.

Corollary 1. If $H_{\Lambda}$ may be written as

$$
H_{\Lambda}=H_{\Lambda}{ }^{0}+\lambda H_{\Lambda}{ }^{1}
$$

where $\mathrm{H}_{A}{ }^{0}$ contains all one- and two-body terms and the latter are all ferromagnetic, the conditions of the theorem will be met for some sufficiently small value of the "perturbation parameter" $\lambda$.

Corollary 2. A sufficient but not necessary condition for the requirements of the theorem to be met is that

$$
U_{E_{x}}(\alpha) \geqq 0
$$

with equality holding only if all spins in $E_{x}$ have the same sign and inequality holding otherwise.

The proof of the theorem proceeds by showing that different equilibrium states (Gibbs distributions) exist at some field value for the two pure boundary conditions. That there is such a field at conditions of strong coupling (low temperature) follows from the solubility of two sets of coupled "integral" equations for the probability of contours with +, respectively -, spins outside, after the manner of Minlos and Sinai [12].

In order to apply the Pirogov-Sinai theorem to the problem of hard core lattice gases it is necessary first to transform them into equivalent spin systems, then to determine the field $h^{*}$, and finally to show that the conditions of the theorem (actually Corollary 2 ) are met. These steps are taken in the following sections.

\section{Equivalent Spin System}

Let us denote the two sublattices of the total system as $\Lambda_{A}$ and $\Lambda_{B}$, so that $\Lambda=\Lambda_{A} \cup \Lambda_{B}$. Throughout this section and the next we assume periodic couplings. For $x \in \Lambda_{A}\left(\Lambda_{B}\right)$ let $F_{x} \subset \Lambda_{B}\left(\Lambda_{A}\right)$ denote the $c$ nearest neighbors of site $x$. If we also denote by $\mu_{A}\left(\mu_{B}\right)$ the chemical potential of a particle at a site of sublattice $A(B)$, we may write the grand partition function as

$$
\Xi_{A B}\left(z_{A}, z_{B}\right)=\sum_{R \subset A_{A}} z_{A}^{|R|} \sum_{S \subset \Lambda_{B} \backslash R^{+}} z_{B}^{|S|}
$$

where $z_{A},_{B}=\exp \left(\beta \mu_{A},{ }_{B}\right)$ and

$$
R^{+}=\bigcup_{x \in R} F_{x} .
$$


It is clear from Eq. (10) that we are using in a basic way the two characteristics of the models under consideration: nearest neighbor exclusions and the existence of only two sublattices. The similarity between this partition function and that of the lattice version of the " $A-B$ " model of Widom and Rowlinson [2] should be obvious. In fact, to convert the present problem to an " $A-B$ " model it is necessary only to identify $\Lambda_{A}$ with $\Lambda_{B}$ and replace $F_{x}$ by $F_{x} \cup\{x\}$.

In any event the previously exploited technique may be used here to sum over configurations of $B$ particles to obtain an equivalent one component lattice gas on lattice $\Lambda_{A}$. This one component lattice gas, however, has short ranged interactions which we write as

$$
H(R)=-\mu|R|-\Phi(R) \varepsilon,
$$

where $\varepsilon$ is a positive constant with dimensions of energy. The onecomponent partition function may then be written as

$$
\Xi(z, \beta)=\exp \left(\beta \varepsilon\left|\Lambda_{B}\right| \sum_{R \subset \Lambda_{A}} \exp [-\beta H(R)],\right.
$$

where the intensive parameters activity $z$ and inverse temperature $\beta$ are related to $z_{A}$ and $z_{B}$ by (see Eq. (4) of Ref. [8])

$$
\begin{gathered}
\beta \varepsilon=\ln \left(1+z_{B}\right) \\
\beta(\mu+c \varepsilon)=\ln z_{A} .
\end{gathered}
$$

We will henceforth discard the analytic factor $\exp \left(\beta \varepsilon\left|\Lambda_{B}\right|\right)$ preceeding the summation in Eq. (13).

The analog potential function $\Phi(R)$ contains many-body terms - up to $c$-body terms - and may be decomposed [8] as

$$
\begin{aligned}
\Phi(R) & =\sum_{\substack{P \subset R \\
|P| \geqq 2}} \varphi_{P} \\
& \equiv \sum_{\substack{P \subset R \\
|P| \geqq 2}}(-)^{P} v_{P}
\end{aligned}
$$

where $v_{P}$ is given by the following prescription: If there is some $x \in \Lambda_{B}$ such that $P \subset F_{x}$, then $v_{P}$ is the number of translates of $P$ contained in $F_{x}$; if there is no such $x$, then $v_{P}=0$. (This applies only for $|P| \geqq 2$.) In equations such as Eq. (15), we use the convention that a set used as an exponent, such as $(-)^{P}$ or $2^{P}$, stands for the cardinality $|P|$ of the set.

The work of Gruber and Merlini [9] contains a careful study of the symmetry characteristics of the original spin system and the equivalent sublattice spin system. There is one point that should be mentioned here by way of contrast. An important conclusion of the Gruber-Merlini approach is a description of the nature of the many-spin interactions in the equivalent sublattice system (derived from an ordinary Ising 
model on an open sublattice). If the original Ising system possesses only nearest neighbor pair interactions, then in zero field the sublattice system (after the partial trace) possesses many-spin interactions of only even order. For the present model this elimination of odd-order interactions does not occur since the lattice gas equivalent of zero field is an infinitely large chemical potential (a consequence of the hard core repulsion). Consequently interactions of odd order occur here as they would for the Ising model with finite interactions and nonvanishing field.

Our equivalent lattice gas may, as always, be interpreted as an Ising spin system with Hamiltonian given by Eq. (1). The general relationship between the coefficients $J_{R}$ and $\varphi_{P},[13]$

$$
J_{R}=\sum_{P \supset R} 2^{-P} \varphi_{P}
$$

was exploited in Ref. [8] to show that

for $|R| \geqq 2$.

$$
J_{R}=2^{-c}(-)^{R} v_{R} \varepsilon
$$

We may also obtain explicit formulas for the one-body terms (magnetic fields) $h=J_{\{x\}}$ and the constant term $J_{\varphi}$. For the former we notice that Eq. (16) for $R=\{x\}$ can be written

$$
J_{\{x\}}=\mu / 2+\varepsilon \sum_{P \subset E_{x}} 2^{-P}(-)^{P} v_{P}\left(|P| / v_{P}\right)
$$

according to the definition of $v_{P}$. Here and below, for $x \in \Lambda_{A}, E_{x}=F_{y}$ where $y \in \Lambda_{B}$ is any one specified nearest neighbor of $x$. We will henceforth use the special notation that $\Sigma^{\prime}$ means a summation over certain sets, restricted to cardinality two or greater. Using the formula (A4) from the Appendix this reduces to

$$
J_{\{x\}}=h=\mu / 2+\varepsilon\left[c / 2-c 2^{-c}\right] .
$$

With periodic boundary connections the constant term $J_{\varphi}$ is proportional to $|\Lambda|$, and $j_{\varphi} \equiv J_{\varphi} /|\Lambda|$ is given by

$$
j_{\varphi}=\mu / 2+\varepsilon \sum_{P \subset E_{x}}^{\prime} 2^{-P}(-)^{P} v_{P} / v_{P}
$$

for any choice of $x$. Using Eq. (A 1) this becomes

$$
j_{\varphi}=\mu / 2+\varepsilon\left[c / 2+2^{-c}-1\right] \text {. }
$$

Equations (17), (19), and (21) define the Ising spin system equivalent to the original hard-core lattice gas. Notice that it is possible to have periodic boundary connections between the spins and still be able to employ special boundary configurations of those spins.

The spin system resides on one of the two sublattices of the lattice in question. Thus if the original lattice is the two-dimensional square 

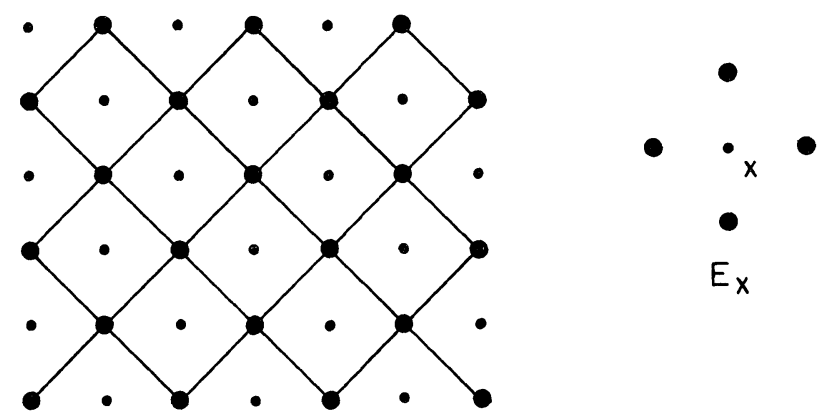

Fig. 1. Hard square lattice gas. The equivalent Ising spin system resides on one of the sublattices, which is also a square lattice. Each "exclusion sphere" $E_{\chi}$ consists of four sites. For any $P \subset E_{x}$ there is a nonvanishing coefficient $J_{P}$ in the Hamiltonian
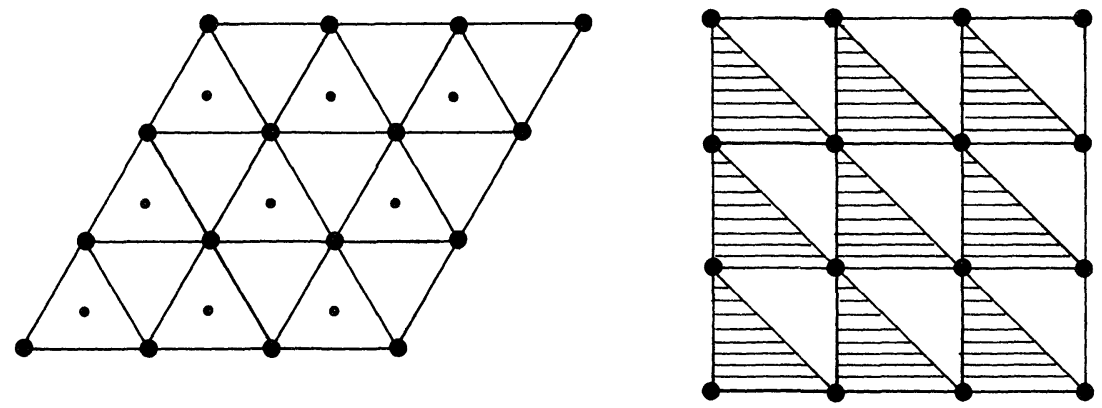

Fig. 2. Hexagonal lattice gas. The sublattice spin system occupies the triangular lattice. Nearest neighbor pair interactions are ferromagnetic $\left(J_{P}>0\right)$. For the three-body terms, operating around half of the triangles, $J_{P}<0$. The Ising system can also be represented on the square lattice, with pair interactions along each bond and three-body interactions around the shaded triangles

lattice, each sublattice is again the square lattice, with interactions among nearest and next nearest neighbor sites (see Fig. 1). The sublattices of the hexagonal lattice are triangular lattices, with two- and three-body forces (Fig. 2). The sublattices of the bodycentered cubic lattice are simple cubic, with interactions of order through eighth (among the eight spins at the corners of the sublattice unit cell).

Both the diamond and the simple cubic lattices have face-centered cubic sublattices - the former with two-, three-, and four-body forces among tetrahedrally disposed sublattice sites, and the latter with interactions among octahedrally arranged sites.

With a reduction of visual symmetry the non-cubic sublattices may be distorted into cubic lattices in the appropriate number of dimensions (with skew interactions), in order to apply the Pirogov-Sinai theorem. 
The triangular sublattice of the hexagonal lattice thus becomes a square lattice, with interactions among spins at $\operatorname{sites}(x, y),(x+1, y)$ and $(x, y+1)-$ see Fig. 2. Similarly the face-centered cubic lattice distorts into a simple cubic lattice with interactions determined by the original lattice. For the diamond lattice, the equivalent spin system has interactions among spins at the sites $(x, y, z),(x+1, y, z),(x, y+1, z),(x, y, z-1)$. For the simple cubic original lattice, six sites interact in the equivalent spin system: $(x, y, z),(x+1, y, z),(x, y+1, z),(x+1, y, z+1),(x, y+1, z+1)$, $(x+1, y+1, z+1)$.

None of the details of the geometry is important, however, for we can show applicability of the Pirogov-Sinai theorem without reference to the geometry.

\section{Critical Field and Contour Energies}

We first compute the energy density of the $(-)$ and the $(+)$ configurations. The former is given by

$$
\begin{aligned}
\frac{H_{\Lambda}(-)}{|\Lambda|} & =j_{\varphi}-J_{\{x\}}+\sum_{P \subset E_{x}}^{\prime}(-)^{P} J_{P} / v_{P} \\
& =\varepsilon\left[(c+1) 2^{-c}-1\right]+\varepsilon 2^{-c} \sum_{P \subset E_{x}}^{\prime} 1 .
\end{aligned}
$$

We have used Eqs. (19) and (21) for the first two terms and Eq. (17) for the last. By virtue of the summation formula (A 3), the entire expression vanishes: $H_{A}(-)=0$. This is correct since the $(-)$ configuration corresponds to the completely empty lattice gas, which has zero energy.

For the $(+)$ configuration we have

$$
\begin{aligned}
\frac{-H_{\Lambda}(+)}{|\Lambda|} & =j_{\varphi}+J_{\{x\}}+\sum_{P \subset E_{x}}^{\prime} J_{P} / v_{P} \\
& =\mu+\varepsilon(c-1)\left(1-2^{-c}\right)+\varepsilon 2^{-c} \sum_{P \subset E_{x}}^{\prime}(-)^{P} \\
& =\mu+\varepsilon(c-1)
\end{aligned}
$$

using formula (A 2). Eliminating $\mu$ in favor of $h=J_{\{x\}}$ we have

$$
\frac{-H_{\Lambda}(+)}{|\Lambda|}=2 h+\varepsilon\left[c 2^{-(c-1)}-1\right]
$$

which we can solve for the critical field $h^{*}$ which makes $H_{\Lambda}(+)=H_{\Lambda}(-)=0$ :

$$
h^{*}=\varepsilon\left(1 / 2-c 2^{-c}\right) \text {. }
$$

According to the development of Pirogov and Sinai, $h^{*}$ is the value of the magnetic field at the phase transition at $T=0$. For small but 
nonzero temperature the critical field value is near $h^{*}$. It should be noted that $h^{*}$ is always positive (since $c>2$ ).

We now turn to the contour energies. Equation (6) for the energy (density) of the finite set $E_{x}$ in the field $h^{*}$ may be written as

$$
-U_{E_{x}}^{\prime}-j_{\varphi}=\left(h^{*} / c\right) \sum_{y \in E_{x}} \sigma_{y}+\sum_{P \subset E_{x}}^{\prime} J_{P} \sigma_{P} / v_{P} .
$$

This is a function of the spin configuration on $E_{x}$, which is conveniently characterized by the set $Q \subset E_{x}$ :

Using Eq. (22) we have

$$
\sigma_{x}= \begin{cases}+1 & x \in Q \\ -1 & x \notin Q\end{cases}
$$

$$
\begin{aligned}
-U_{E_{x}}^{\prime}-j_{\varphi}= & \left(1 / 2-c 2^{-c}\right)[2(q / c)-1] \varepsilon \\
& +2^{-c} \varepsilon \sum_{P \subset E_{x}}^{\prime} \sigma_{P}(Q)
\end{aligned}
$$

where

$$
\sigma_{P}(Q)=(-)^{P \cap Q}
$$

and $q=|Q|$.

It is shown in the Appendix [Eq. (A 7)] that

This means that

$$
\sum_{P \subset E_{x}}^{\prime} \sigma_{P}(Q)= \begin{cases}2 q-(c+1) & q \neq 0 \\ 2^{c}-(c+1) & q=0 .\end{cases}
$$

$$
\begin{aligned}
U_{E_{x}}^{\prime} & = \begin{cases}\varepsilon(1-q / c) & q \neq 0 \\
0 & q=0\end{cases} \\
& \equiv U_{E_{x}},
\end{aligned}
$$

since $U_{E_{x}}^{\prime}=0$ for $q=c$ or $q=0$. Since $0 \leqq q \leqq c$ this last result establishes the conditions of Corollary 2.

\section{Discussion}

The proof is now complete that there is at least one magnetic field value at which the equivalent Ising spin system has more than one (boundary-controlled) equilibrium state, at sufficiently low temperature. By virtue of Eqs. (19) and (14) the corresponding statement for the original hard sphere lattice gas is that for sufficiently large activity $z_{B}$ on one sublattice there is as least one value of the activity $z_{A}$ on the other at which more than one equilibrium state exists. We believe that "at least" could be replaced by "exactly" and the statement would still be true, but that is not proven.

The presumption that the region of nonuniqueness is described completely by a condition of the form $z_{A}=z_{B} \geqq z_{0}$ is tantamount to an 
explicit expression for the temperature dependence of the transition field value whose existence is guaranteed by the Pirogov-Sinai theorem. We need only set $z_{A}=z_{B}$ in Eqs. (14) and eliminate $\mu$ in favor of $h$ by Eq. (19). We thus obtain

$$
2 \beta h=\ln \left(e^{\beta \varepsilon}-1\right)-\beta \varepsilon c 2^{-(c-1)} .
$$

This has the low temperature limit

$$
\lim _{\beta \rightarrow \infty} h / \varepsilon=1-c 2^{-(c-1)}
$$

but we do not know the critical temperature above which Eq. (24) is simply a symmetry line but not a phase transition line.

\section{Appendix}

We derive here several useful summation formulas. We let $E$ be an arbitrary set of $c$ elements, $c \geqq 0$. We first calculate

$$
\begin{aligned}
G(E ; x) & \equiv \sum_{P \subset E}^{\prime}(-x / 2)^{P} \\
& =(1-x / 2)^{c}+c x / 2-1
\end{aligned}
$$

from the binomial theorem. Special cases are conveniently written explicity: For $x=1$ we have

$$
\sum_{P \subset E}^{\prime}(-)^{P} 2^{-P}=c / 2+2^{-c}-1 .
$$

By setting $x=2$ we have

$$
\sum_{P \subset E}^{\prime}(-)^{P}=c-1,
$$

which, however, is not valid for $c=0$. By letting $x=-2$ we have

$$
\sum_{P \subset E}^{\prime} 1=2^{c}-1-c .
$$

The derivative $(\partial G / \partial x)_{x=1}$ gives us

$$
\sum_{P \subset E}^{\prime}(-)^{P} 2^{-P}|P|=c / 2-c 2^{-c} .
$$

Finally we consider the function

$$
S(Q)=\sum_{P \subset E}^{\prime} \sigma_{P}(Q)=\sum_{\substack{P_{2} \subset E \backslash Q \\\left|P_{1}\right|+\left|P_{2}\right| \geqq Q}} \sum_{\substack{1 \\ P_{1} \mid}}(-)^{P_{1}},
$$


where $\sigma_{P}(Q)=(-)^{P \cap Q}$. Assuming $q=|Q|>0$ we expand the right-hand side to obtain

$$
S(Q)=\sum_{P_{2} \subset E \backslash Q}^{\prime} 1-q \sum_{\substack{P_{2} \subset E \backslash Q \\\left|P_{2}\right| \geqq 1}} 1+\sum_{P_{2} \subset E \backslash Q} \sum_{P_{1} \subset Q}^{\prime}(-)^{P_{1}} .
$$

Equation (A3) is easily modified to give

$$
\sum_{\substack{P \subset E \\|P| \geqq 1}} 1=2^{c}-1, \sum_{P \subset E} 1=2^{c} .
$$

With the help of (A2) we have then

$$
S(Q)= \begin{cases}2 q-c-1 & q \neq 0 \\ 2^{c}-c-1 & q=0,\end{cases}
$$

where the $q=0$ case follows directly from Eqs. (A 5) and (A 3).

Acknowledgement. Many helpful discussions with Professor J. L. Lebowitz are gratefully acknowledged, as is the hospitality of Belfer Graduate School of Science, Yeshiva University, where most of this work was done.

Note Added in Proof. It has recently been shown [Heilmann, O.J.: Commun. math. Phys. 36, 91-114 (1974)] how the Peierls contour approach can be adapted to lattice problems having two sublattices related by reflection rather than by translation. The hexagonal and diamond lattices fall in this category.

\title{
References
}

1. Griffiths, R. B.: Phase transitions and critical phenomena, Vol. 1, Chapter 2; Domb, C., Green, M.S. (eds.), New York: Academic Press 1972. Gallavotti, G.: Riv. Nuovo Cimento 2, 133 (1972)

2. Widom, B., Rowlinson, J. S. : J. Chem. Phys. 52, 1670 (1970); Runnels, L. K., Lebowitz, J.L.: J. Math. Phys. 15, 1712 (1974)

3. Dobrushin, R.L.: Funct. Anal. Applic. 2, 302 (1968)

4. Heilmann, O. J.: J. Stat. Phys. 9, 23 (1974)

5. Runnels, L. K., Combs, L. L., Salvant, J.P.: J. Chem. Phys. 47, 4015 (1967)

6. Gaunt, D.S.: J. Chem. Phys. 46, 3237 (1967)

7. Pirogov, S.A., Sinai, Y. G.: Funct. Anal. (Russian), No. 1, 1974

8. Runnels, L. K.: J. Math. Phys. 15, 984 (1974)

9. Gruber, C., Merlini, D.: Physica 67, 308 (1973)

10. Fisher, M.E.: Phys. Rev. 113, 969 (1959)

11. Widom, B.: J. Chem. Phys. 46, 3324 (1967); Neece, G. : J. Chem. Phys. 47, 4112 (1967); Clark, R. K.: J. Chem. Phys. 48, 741 (1968). See also Mulholland, G. W., Rehr, J.J.: J. Chem. Phys. 60, 1297 (1974)

12. Minlos, R.A., Sinai, J.G.: Trans. Moscow Math. Soc. 17, 237 English (1967)

13. Fortuin,C.M., Kasteleyn,P.W., Ginibre, J.: Commun. math. Phys. 22, 89 (1971)

Communicated by G. Gallavotti

\author{
L. K. Runnels \\ Department of Chemistry \\ Lousiana State University \\ Baton Rouge, Louisiana 70803, USA
}

